\title{
Dear Author,
}

Please use this PDF proof to check the layout of your article. If you would like any changes to be made to the layout, you can leave instructions in the online proofing interface.

Making your changes directly in the online proofing interface is the quickest, easiest way to correct and submit your proof. Please note that changes made to the article in the online proofing interface will be added to the article before publication, but are not reflected in this PDF proof.

If you would prefer to submit your corrections by annotating the PDF proof, please download and submit an annotatable PDF proof by following this link:

https://rscweb.proofcentral.com/en/offline.html? token $=3 \mathrm{dc} 58 \mathrm{e} 2 \mathrm{a} 6 \mathrm{a} 6 \mathrm{dfb} 4 \mathrm{~b} 186573 \mathrm{a} 3 \mathrm{dde} 45 \mathrm{f} 8 \mathrm{~b}$ 
We have presented the graphical abstract image and text for your article below. This briefly summarises your work, and will be presented with your article online.

1

lodide mediated reductive decomposition of diazonium salts: towards mild and efficient covalent functionalization of surface-supported graphene

Yuanzhi Xia, Cristina Martin, Johannes Seibel, Samuel Eyley, Wim Thielemans, Mark van der Auweraer, Kunal Mali* and Steven De Feyter*

We present an efficient and straightforward covalent functionalization protocol for modification of surface supported graphene and graphite using aqueous reagents.

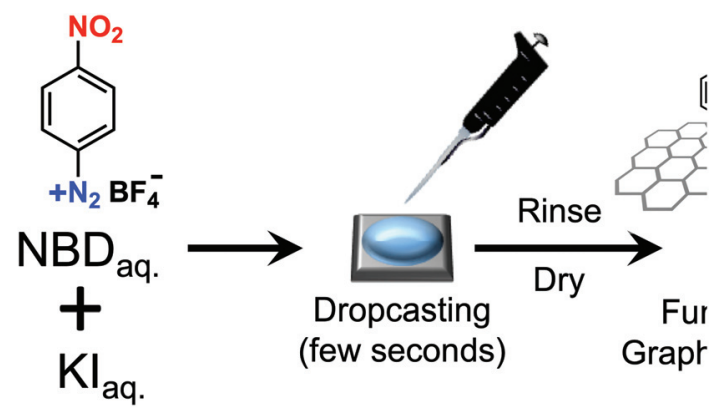

Please check this proof carefully. Our staff will not read it in detail after you have returned it.

Please send your corrections either as a copy of the proof PDF with electronic notes attached or as a list of corrections. Do not edit the text within the PDF or send a revised manuscript as we will not be able to apply your corrections. Corrections at this stage should be minor and not involve extensive changes.

Proof corrections must be returned as a single set of corrections, approved by all co-authors. No further corrections can be made after you have submitted your proof corrections as we will publish your article online as soon as possible after they are received.

Please ensure that:

- The spelling and format of all author names and affiliations are checked carefully. You can check how we have identified the authors' first and last names in the researcher information table on the next page. Names will be indexed and cited as shown on the proof, so these must be correct.

- Any funding bodies have been acknowledged appropriately and included both in the paper and in the funder information table on the next page.

- All of the editor's queries are answered.

- Any necessary attachments, such as updated images or ESI files, are provided.

Translation errors can occur during conversion to typesetting systems so you need to read the whole proof. In particular please check tables, equations, numerical data, figures and graphics, and references carefully.

Please return your final corrections, where possible within $\mathbf{4 8}$ hours of receipt following the instructions in the proof notification email. If you require more time, please notify us by email to nanoscale@rsc.org. 


\section{Funding information}

Providing accurate funding information will enable us to help you comply with your funders' reporting mandates. Clear acknowledgement of funder support is an important consideration in funding evaluation and can increase your chances of securing funding in the future.

We work closely with Crossref to make your research discoverable through the Funding Data search tool (http://search. crossref.org/funding). Funding Data provides a reliable way to track the impact of the work that funders support. Accurate funder information will also help us (i) identify articles that are mandated to be deposited in PubMed Central (PMC) and deposit these on your behalf, and (ii) identify articles funded as part of the CHORUS initiative and display the Accepted Manuscript on our web site after an embargo period of 12 months.

Further information can be found on our webpage (http://rsc.li/funding-info).

\section{What we do with funding information}

We have combined the information you gave us on submission with the information in your acknowledgements. This will help ensure the funding information is as complete as possible and matches funders listed in the Crossref Funder Registry.

If a funding organisation you included in your acknowledgements or on submission of your article is not currently listed in the registry it will not appear in the table on this page. We can only deposit data if funders are already listed in the Crossref Funder Registry, but we will pass all funding information on to Crossref so that additional funders can be included in future.

\section{Please check your funding information}

The table below contains the information we will share with Crossref so that your article can be found via the Funding Data search tool. Please check that the funder names and grant numbers in the table are correct and indicate if any changes are necessary to the Acknowledgements text.

\begin{tabular}{|l|l|l|l|}
\hline Funder name & $\begin{array}{l}\text { Funder's main country } \\
\text { of origin }\end{array}$ & $\begin{array}{l}\text { Funder ID } \\
\text { (for RSC use only) }\end{array}$ & Award/grant number \\
\hline $\begin{array}{l}\text { Fonds Wetenschappelijk } \\
\text { Onderzoek }\end{array}$ & Belgium & 501100003130 & $\begin{array}{l}30489208 \\
12 J 1719 \mathrm{~N} / 12 J 1716 \mathrm{~N}\end{array}$ \\
\hline $\begin{array}{l}\text { FP7 Ideas: European Research } \\
\text { Council }\end{array}$ & European Union & 100011199 & $\begin{array}{l}\text { ERC Grant Agreement } \\
340324\end{array}$ \\
\hline China Scholarship Council & China & 501100004543 & Unassigned \\
\hline $\begin{array}{l}\text { H2020 Marie Skłodowska-Curie } \\
\text { Actions }\end{array}$ & European Union & 100010665 & 789865-EnSurf \\
\hline KU Leuven & Belgium & 501100004040 & 3E180424 \\
\hline
\end{tabular}

\section{Researcher information}

Please check that the researcher information in the table below is correct, including the spelling and formatting of all author names, and that the authors' first, middle and last names have been correctly identified. Names will be indexed and cited as shown on the proof, so these must be correct.

If any authors have ORCID or ResearcherID details that are not listed below, please provide these with your proof corrections. Please ensure that the ORCID and ResearcherID details listed below have been assigned to the correct author. Authors should have their own unique ORCID iD and should not use another researcher's, as errors will delay publication.

Please also update your account on our online manuscript submission system to add your ORCID details, which will then be automatically included in all future submissions. See here for step-by-step instructions and more information on author identifiers.

\begin{tabular}{|l|l|l|l|}
\hline First (given) and middle name(s) & Last (family) name(s) & ResearcherID & ORCID iD \\
\hline Yuanzhi & Xia & & \\
\hline Cristina & Martin & &
\end{tabular}




\begin{tabular}{|l|l|l|l|}
\hline Johannes & Seibel & & \\
\hline Samuel & Eyley & & 0000-0002-1929-8455 \\
\hline Wim & Thielemans & A-4506-2010 & 0000-0003-4451-1964 \\
\hline Mark & van der Auweraer & & \\
\hline Kunal & Mali & & 0000-0002-9938-6446 \\
\hline Steven & De Feyter & K-7245-2014 & 0000-0002-0909-9292 \\
\hline
\end{tabular}




\section{Queries for the attention of the authors}

\section{Journal: Nanoscale Paper: d0nr03309j}

Title: lodide mediated reductive decomposition of diazonium salts: towards mild and efficient covalent functionalization of surface-supported graphene

For your information: You can cite this article before you receive notification of the page numbers by using the following format: (authors), Nanoscale, (year), DOI: 10.1039/d0nr03309j.

Editor's queries are marked like this Q1, Q2, and for your convenience line numbers are indicated like this $5,10,15, \ldots$

Please ensure that all queries are answered when returning your proof corrections so that publication of your article is not delayed.

\begin{tabular}{|c|c|c|}
\hline $\begin{array}{l}\text { Query } \\
\text { Reference }\end{array}$ & Query & Remarks \\
\hline Q1 & $\begin{array}{l}\text { Funder details have been incorporated in the funder table using } \\
\text { information provided in the article text. Please check that the } \\
\text { funder information in the table is correct. }\end{array}$ & \\
\hline Q2 & $\begin{array}{l}\text { Please confirm that the spelling and format of all author names } \\
\text { is correct. Names will be indexed and cited as shown on the } \\
\text { proof, so these must be correct. No late corrections can be } \\
\text { made. }\end{array}$ & \\
\hline Q3 & $\begin{array}{l}\text { In the sentence beginning "Fig. } 4 \mathrm{e} \text { shows that the layer..." there } \\
\text { is a citation to Fig. } 4 \mathrm{e} \text {, however Fig. } 4 \text { does not appear to } \\
\text { contain a part (e). Please resupply the figure (preferably as a TIF } \\
\text { file at } 600 \text { dots per inch) or amend the text accordingly. }\end{array}$ & \\
\hline Q4 & $\begin{array}{l}\text { The award/grant numbers in the article text and in the funder } \\
\text { information do not match, please check and correct as } \\
\text { necessary. }\end{array}$ & \\
\hline Q5 & $\begin{array}{l}\text { Ref. 3: Please check that the last name for the 9th author is } \\
\text { displayed correctly. }\end{array}$ & \\
\hline Q6 & $\begin{array}{l}\text { Ref. 20: Please check that the last name for the } 5 \text { th author is } \\
\text { displayed correctly. }\end{array}$ & \\
\hline Q7 & $\begin{array}{l}\text { Ref. 27: Please check that the last name for the } 4 \text { th author is } \\
\text { displayed correctly. }\end{array}$ & \\
\hline
\end{tabular}




\title{
lodide mediated reductive decomposition of diazonium salts: towards mild and efficient covalent functionalization of surface-supported graphene $\uparrow$
}

Cite this: DOI: 10.1039/d0nr03309

\author{
Yuanzhi Xia, ${ }^{a}$ Cristina Martin, ${ }^{\text {b,c }}$ Johannes Seibel, ${ }^{a}$ Samuel Eyley, (D) ${ }^{c}$ \\ Wim Thielemans, (D) ${ }^{c}$ Mark van der Auweraer, ${ }^{a}$ Kunal Mali (D) ${ }^{a}$ and \\ Steven De Feyter (D)*a
} of the chemically modified material, but also because it brings fundamental insight into the chemistry of graphene. Thus, strategies that yield chemically modified graphene with densely grafted films of aryl groups via simple experimental protocols have been the focus of intense research. Here we report a mild, straightforward and efficient approach to graphene/graphite functionalization using iodide mediated reductive dediazoniation of aryldiazonium salts. The experimental protocol employs aqueous solutions of the reagents. The reaction proceeds rapidly at room temperature without the need of any environmental or electrochemical control. The covalently modified surfaces were characterized at the nanometer scale using a combination of complementary surface analytical techniques. The degree of covalent functionalization, and the morphology, as well as the thickness of the grafted films were studied at the molecular level using Raman spectroscopy and scanning probe microscopy, respectively. Furthermore, solution phase UV-Vis spectroscopy was employed to understand the mechanistic aspects. This work demonAccepted 26th May 2020

DOI: $10.1039 /$ dOnr03309j strates a facile and scalable covalent modification method compatible for both bulk and monolayer rsc.li/nanoscale functionalization of graphene.

\section{Introduction}

Covalent functionalization of graphene is an emerging area of research. It involves covalent attachment of organic moieties onto the basal plane of graphene. The covalent bond formation between graphene and the addend proceeds via $\mathrm{sp}^{2}$ to $\mathrm{sp}^{3}$ rehybridization of the carbon atom of the graphene lattice to which the organic moiety is added. Such chemical modification of graphene is being widely explored as it offers opportunities to improve the processability of the material which is otherwise insoluble in common solvents. It also allows synthesis of composite materials wherein the unique properties of

\footnotetext{
${ }^{a}$ Department of Chemistry, Division of Molecular Imaging and Photonics, KU Leuven, Celestijnenlaan 200F, B-3001Leuven, Belgium.E-mail: kunal.mali@kuleuven.be, steven.defeyter@kuleuven.be

${ }^{b}$ Departamento de Quimica Física, Facultad de Farmacia, Universidad de Castilla-La Mancha, 02071 Albacete, Spain

${ }^{c}$ Department of Chemical Engineering, Sustainable Materials Lab, KU Leuven,

Campus Kulak Kortrijk, Etienne Sabbelaan 53, 8500 Kortrijk, Belgium

$\dagger$ Electronic supplementary information (ESI) available. See DOI: 10.1039/ d0nr03309j
}

graphene can be combined with those of electro- and photoactive organic moieties. The generation of $\mathrm{sp}^{3}$ defects within the $\mathrm{sp}^{2}$ lattice of covalently functionalized graphene leads to modification of its band structure thereby allowing modulation of its electronic and optical properties. ${ }^{1}$

Given the chemical inertness of its basal plane, the covalent functionalization of graphene can only be achieved using reactive intermediates such as free radicals, carbenes, nitrenes and arynes. These species form covalent adducts with graphene via free radical addition, $\mathrm{CH}$ insertion or cycloaddition reactions. ${ }^{2-4}$ Amongst the different strategies, diazonium chemistry has been particularly popular for the covalent attachment of aryl groups to the basal plane of graphene. The usefulness of aryldiazonium salts for covalent modification of carbon surfaces has been known since the early 1990s. ${ }^{5}$ Typically, aryl diazonium salts are electrochemically reduced in the vicinity of carbon surfaces which act as cathodes. The reductive decomposition of aryldiazonium salts yields aryl radicals through the loss of nitrogen. The radicals then attach to the carbon (graphene) surface. ${ }^{6}$ Aryldiazonium salts are also known to react spontaneously with carbon (graphene) surfaces. The spontaneous addition preferably occurs at the edges 
of graphene as the basal plane is rather unreactive. ${ }^{7}$ Covalent functionalization of graphene using spontaneous reaction has been reported in a few cases, however since the reaction proceeds sluggishly, long reaction times are needed. ${ }^{8}$

Diazonium chemistry-based covalent functionalization of graphene can be classified into two categories depending on the topicity of the radical addition reaction. For graphene supported by an inert solid substrate, radical attack is possible only from one side, leading to supratopic addition. On the other hand, for graphene sheets suspended in solution, radical attack can occur from both sides of the basal plane leading to antaratopic addition. ${ }^{9}$ Furthermore, the experimental protocols used for surface-supported and solution-suspended graphene differ drastically. One of the most popular and efficient routes for covalent functionalization of solution suspended graphene involves the use of graphite intercalation compounds (GICs) also known as graphenides. ${ }^{10}$ GICs are obtained via reduction of graphite using alkaline metals such as $\mathrm{Na}$ or $\mathrm{K}$ and consist of sheets of graphene separated by $\mathrm{Na}$ or $\mathrm{K}$ ions. Such intercalation renders the material nucleophilic thereby 'activating' it for reaction with electrophiles such as diazonium salts. Furthermore, intercalation also introduces coulombic repulsion between the sheets allowing facile exfoliation in appropriate (organic) solvents. Given that GICs are pyrophoric materials and thus ignite spontaneously when they contact water or oxygen, the whole process is carried out under strictly inert conditions. The success of the approach critically depends on the exclusion of oxygen and water $(<0.1 \mathrm{ppm})$ from the reaction mixture. ${ }^{11}$ Very recently, $\mathrm{Na} / \mathrm{K}$ alloy-based activation procedure was applied for modification of graphene supported by $\mathrm{Si} / \mathrm{SiO}_{2}$ for achieving reversible covalent patterning of graphene. ${ }^{12}$

The covalent functionalization of graphene supported by solid substrates ${ }^{13}$ is often carried out either using spontaneous reaction with diazonium salts ${ }^{14}$ or via their electrochemical reduction. ${ }^{15}$ For spontaneous reaction, the substrates are immersed in a solution of diazonium salts for several hours. The density of surface attachment of aryl groups obtained via spontaneous reaction with graphene is often low. The electrochemical method on the other hand, yields densely grafted surfaces due to the 'activated' nature of the process. Graphene itself acts as a cathode providing electrons to the diazonium cations at the interface. Single or few layered graphene supported by substrates such as $\mathrm{SiC}$, metals and $\mathrm{SiO}_{2}$ is functionalized using this approach. Highly oriented pyrolytic graphite (HOPG) can be used as a model substrate for such studies. Despite the lower reactivity of HOPG compared to that of single layered graphene, relatively high grafting densities can be readily achieved using the electrochemical method. Using appropriate design of aryldiazonium salts, we have recently demonstrated precise control over the density of covalent grafting onto the surface of HOPG. ${ }^{16-19}$

Both the approaches described above have progressed substantially in the past few years. In contrast to the bulk approach, which allows functionalization on a gram scale, the electrochemical approach is limited to surface functionali- zation with areas extending only up to a few square centimetres. The bulk strategies, however, often involve the use of pyrophoric materials with strict environmental control. Despite using the activated forms of graphite, namely the GICs, the functionalization reactions often run for several hours at elevated temperatures. Covalently functionalized graphene obtained using the bulk approach is characterized using Raman spectroscopy, thermogravimetric analysis (TGA), FT-IR, gas chromatography (GC), mass spectrometry (MS) etc. Recently an advanced characterization tool that combines TGA with FT-IR/GC/MS has been employed for comprehensive characterization of functionalized graphene. ${ }^{11}$ The analytical techniques listed above provide ensemble averaged data and thus the information on the local nanometre scale inhomogeneities is often lost. Surface-supported functionalized graphene, however, can be readily characterized at the nanometre scale using state-of-the-art scanning probe methods, namely atomic force- (AFM) and scanning tunnelling microscopy (STM) bringing atomic ${ }^{20}$ and molecular level insight into the covalent functionalization process. ${ }^{16,17}$

In this contribution, we report a mild, facile and efficient approach towards covalent functionalization of graphene and graphite using iodide mediated reductive dediazoniation of diazonium salts. The experimental protocol employs aqueous solutions of the reagents. The functionalization proceeds rapidly at room temperature (few seconds to few minutes) without requiring any environmental or electrochemical control. We demonstrate that by systematic variation of the reaction time, the solution concentration and the molar ratio of the two reagents in solution, it is possible to control the density of covalently attached aryl groups. The functionalized samples were characterized using STM, AFM and Raman spectroscopy, which allows correlation between the film morphology, the film thickness and the density/efficiency of covalent grafting, respectively. The chemical composition of the modified layers was assessed using X-ray photoelectron spectroscopy (XPS). Furthermore, using UV-Vis spectroscopy in solution, we have attempted to deconvolute the mechanistic aspects of the functionalization process. The mild experimental approach presented here is compatible for both bulk and monolayer functionalization.

\section{Results and discussion}

Fig. 1a shows a schematic of the covalent functionalization protocol used in this work. The experiment involves mixing of aqueous solutions of the diazonium salt and KI, immediately followed by drop casting of the solution mixture onto the basal plane of graphene (Fig. 1b). The reaction can then be terminated by washing the surface repeatedly with deionized water and ethanol. The sample is then dried under the stream of argon. The as-prepared substrates were then characterized using Raman, STM, AFM and XPS. 4-Nitrobenzene diazonium tetrafluoroborate (NBD) was chosen as a source of aryl radicals 
a) functionalization experiments. molecule. as it is an archetypal diazonium salt used in covalent

This strategy is inspired from the well-established use of diazonium salts for aryl-aryl couplings. ${ }^{21}$ Iodide induced dediazoniation of aryl diazonium salts has been reported to provide quantitative yields of biaryls. KI was used as a source of iodide ions which serve as reducing agents. ${ }^{22}$ We view such coupling reactions as an opportunity towards the arylation of graphene which can be considered as a giant aromatic

\section{Functionalization of single layered graphene (SLG)}

Evidence for covalent grafting: Raman spectroscopy. Raman spectroscopy, which has emerged as an indispensable tool for the characterization of covalently functionalized graphene $\mathrm{e}^{23}$ was used as the primary analytical tool for evaluating the efficiency of functionalization. The primary Raman spectrum of graphene consists of three main bands: the $G$ band $\left(\sim 1580 \mathrm{~cm}^{-1}\right)$, the $\mathrm{D}$ band $\left(\sim 1336 \mathrm{~cm}^{-1}\right)$ and the $2 \mathrm{D}$ band $\left(\sim 2680 \mathrm{~cm}^{-1}\right)$ when the samples are excited using a $633 \mathrm{~nm}$ laser source. The D band, which is the signature of defects in the lattice, is forbidden in pristine graphene, and thus serves as a reliable indicator of crystallographic quality. ${ }^{24}$ The integrated intensity ratio of the $\mathrm{D}$ - and the G-bands $I_{\mathrm{D}} / I_{\mathrm{G}}$ was used as a measure of the density of the defects on the graphene/ graphite lattice and thus served as a standard for the evaluation of the degree of covalent functionalization.

Fig. 2a shows representative Raman spectra of pristine and NBD functionalized single layered graphene (SLG) on $\mathrm{SiO}_{2}$ $\left(\mathrm{SLG} / \mathrm{SiO}_{2}\right)$. The Raman spectrum of pristine graphene shows a prominent G-band at $\sim 1600 \mathrm{~cm}^{-1}$ and a 2D-band at $\sim 2643 \mathrm{~cm}^{-1}$ whereas the D-band is barely discernible. Upon covalent functionalization with NBD using KI, a prominent D-band at $\sim 1330 \mathrm{~cm}^{-1}$ appeared which was accompanied by the $\mathrm{D}^{*}$ band at $1615 \mathrm{~cm}^{-1}$. The appearance of the D-band clearly demonstrates conversion of the $\mathrm{sp}^{2}$ hybridized atoms of the graphene lattice into $\mathrm{sp}^{3}$ carbon atoms. The intensity of the $2 \mathrm{D}$ band was found to be reduced after functionalization. The $I_{\mathrm{D}} / I_{\mathrm{G}}$ ratio for NBD functionalized graphene was found to be $3.0 \pm 0.5$. Note that this value is amongst the highest reported for surface-supported covalently modified graphene samples (see Table S1 in the ESI $\uparrow$ for comparison). Given its simplicity, the high values of $I_{\mathrm{D}} / I_{\mathrm{G}}$ ratio obtained here clearly highlight the superiority of our functionalization approach. ${ }^{12}$

Since the reactivity of graphene is known to be influenced by the substrate on which it resides, ${ }^{25}$ the efficiency of the functionalization approach was tested using SLG supported by other substrates such as $\mathrm{Cu}(\mathrm{SLG} / \mathrm{Cu})$ and $\mathrm{Au}(\mathrm{SLG} / \mathrm{Au})$. Table 1 provides the $I_{\mathrm{D}} / I_{\mathrm{G}}$ ratios for the different types of graphene samples modified in this work. The high $I_{\mathrm{D}} / I_{\mathrm{G}}$ values obtained for NBD modified SLG/Cu and SLG/Au clearly show that the functionalization approach is universal and can be applied to different types of graphene samples.

Chemical composition: XPS analysis. The chemical composition of the covalently modified SLG/Si samples was examined in detail using XPS. Survey spectra of the samples (Fig. S3 in the ESI $\dagger$ ) revealed the expected carbon, nitrogen, oxygen and silicon signals indicating the presence of nitrophenyl groups on the graphene surface. Trace levels of iodine were also recorded which possibly represent residual iodine originated during the covalent modification process (vide infra).

Fig. $2 \mathrm{~b}$ and $\mathrm{c}$ shows the comparison between the highresolution $\mathrm{C}$ 1s spectrum of pristine and NBD modified graphene, respectively. The $\mathrm{C} 1 \mathrm{~s}$ spectrum of the pristine graphene sample consists of a narrow asymmetric peak at 284.1 $\mathrm{eV}$ corresponding to the $\mathrm{sp}^{2}$ carbon atoms in graphene lattice $(\mathrm{C}=\mathrm{C})$. A single higher binding energy peak at $286.2 \mathrm{eV}$ could be ascribed to a small concentration of oxidized carbon $(\mathrm{C}-\mathrm{O})$ in the graphene sample, while the remainder of the intensity in this sample is due to shake-up effects $\left(\pi^{*} \leftarrow \pi\right)$. No evidence of $\mathrm{C}-\mathrm{C}$ environments could be found in the pristine graphene sample. The $\mathrm{C}=\mathrm{C}$ peak for graphene was found to be shifted to slightly higher binding energy $(284.2 \mathrm{eV})$ for the covalently modified graphene sample. While this value is well-within the 

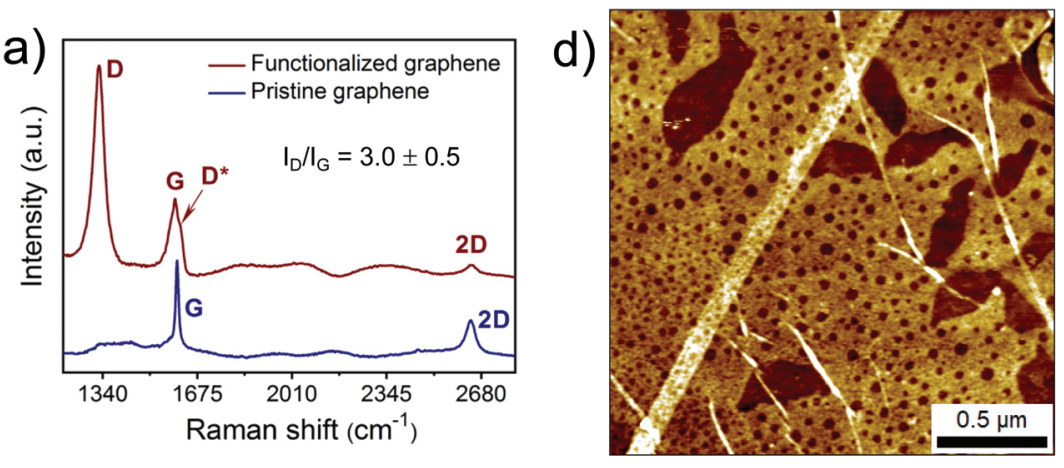

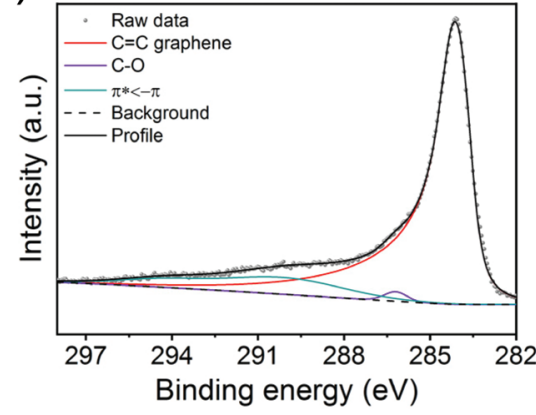

b) XPS C 1s: Pristine

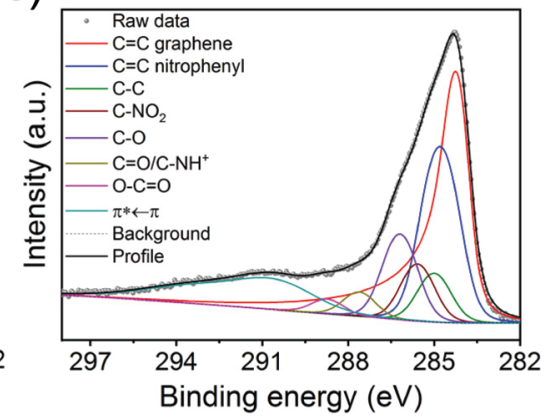

C) XPS C 1s: Functionalized

10

Morphology of the grafted films: AFM. The morphology of

Table $1 I_{D} / l_{G}$ values for different types of graphene samples and HOPG. Reaction conditions: $[\mathrm{NBD}]=10 \mathrm{mM},[\mathrm{KI}]=10 \mathrm{mM}, t=10 \mathrm{~min}$. For representative Raman spectra of functionalized SLG/Cu and SLG/Au, see Fig. S2 in the ESI $\dagger$

\begin{tabular}{lllll}
\hline Sample & $\mathrm{SLG} / \mathrm{SiO}_{2}$ & $\mathrm{SLG} / \mathrm{Cu}$ & $\mathrm{SLG} / \mathrm{Au}$ & $\mathrm{HOPG}$ \\
\hline$I_{\mathrm{D}} / I_{\mathrm{G}}$ & $3.0 \pm 0.5$ & $3.0 \pm 0.4$ & $2.5 \pm 0.5$ & $0.05 \pm 0.01$
\end{tabular}

reported range of graphene binding energies in the literature, the binding energies of graphene are known to be substrate dependent due to charge transfer/screening between the substrate and the graphene layer. ${ }^{26}$ We therefore postulate that the shift in $\mathrm{C}=\mathrm{C}$ binding energy for the NBD modified sample arises due to the changes in the interactions between the graphene layer and the underlying substrate caused by the introduction of the $\mathrm{sp}^{3}$ defects in the graphene lattice. Given that Raman spectroscopy data provided earlier irrefutably confirms the formation of the $\mathrm{sp}^{3}$ defects, this hypothesis is not unreasonable. In addition to the graphene $\mathrm{C}=\mathrm{C}$ peak, environments corresponding to $\mathrm{C}=\mathrm{C}$ from nitrophenyl species $(284.8 \mathrm{eV})$, $\mathrm{C}-\mathrm{C}$ graphene defects (grafting site, $285 \mathrm{eV}$ ) and $\mathrm{C}-\mathrm{NO}_{2}(285.6$ $\mathrm{eV}$ ) were found as expected. Comparison of the intensities of the $\mathrm{C}-\mathrm{NO}_{2}$ and $\mathrm{C}-\mathrm{C}$ peaks gave a ratio of 1.21 : 1 , which is suggestive of multilayer formation on the surface of graphene (see Table $\mathrm{S} 2$ in the ESI $\dagger$ ). For the analysis of the $\mathrm{N}$ 1s spectra see Fig. S4 and Table S3 in the ESI. $\dagger$ the covalently grafted films was characterized using AFM. Fig. 2d shows a representative AFM height image of the NBD modified $\mathrm{SLG} / \mathrm{SiO}_{2}$ which shows a covalent film with areas of lower topographical height scattered throughout the surface. These so-called 'nanocorrals' ${ }^{27}$ are a defining feature of the iodide mediated functionalization strategy. The thickness of the films was found to be $\sim 2 \mathrm{~nm}$ (Fig. S5 in the ESI $\dagger$ ). Given that a monolayer film consisting of single nitrophenyl units standing orthogonal to the surface is expected to show a thickness of $\sim 0.8 \mathrm{~nm}$, we conclude that the covalent film formed under the given experimental conditions consists of a multilayered structure. This observation is in line with the known behaviour of NBD wherein the nitrophenyl radicals attack the surface-attached nitrophenyl groups thereby promoting dendritic polyaryl growth (see Fig. 6a). ${ }^{16,28}$

In order to thoroughly examine the covalent grafting process under varied experimental conditions, we employed HOPG as a model substrate since it offers atomically flat terraces of graphene extending several micrometres. Contamination-free graphene terraces can be readily obtained by simple mechanical exfoliation. Furthermore, similar to graphene, the formation of $\mathrm{sp}^{3}$ defects in the basal plane of graphite is readily discernible from the appearance of the $\mathrm{D}$ band in the Raman spectrum. An important difference however, is in the relative intensities of the peaks. The G peak in graphite for example, is obviously more intense than that in 
graphene. It readily follows that the $I_{\mathrm{D}} / I_{\mathrm{G}}$ ratios are typically much lower for functionalized HOPG compared to SLG substrates. Lastly, the atomically flat nature of the HOPG substrates allows characterization of the grafted films at the single molecule level using STM which is somewhat challenging for graphene samples. The dependence of covalent grafting density on the reaction time, the concentration of reagents and their mole ratio was followed using Raman spectroscopy and scanning probe microscopy, which allow monitoring of the efficiency of covalent grafting vis-à-vis nanoscale surface morphology. As described below, this exercise allowed us to obtain molecular level insight into how fast the aryl radicals are produced and where they react during the interfacial grafting process.

\section{Functionalization of HOPG}

Influence of the reaction time. Fig. 3a shows the evolution of the Raman spectra of functionalized HOPG as a function of reaction time at given concentrations of NBD and KI and at a constant mole ratio (also see Fig. S6 in the ESI†े). As evident from the spectra, the D-band appears even for samples with reaction times as short as 5 seconds and continues to increase in intensity at longer reaction times and then remains constant. In order to clearly understand the changes in the density of covalently attached aryl units, average $I_{\mathrm{D}} / I_{\mathrm{G}}$ values were plotted as a function of the reaction time. Fig. $3 \mathrm{~b}$ reveals that the density of $\mathrm{sp}^{3}$ defects first increases rapidly with increasing reaction time and then reaches a plateau after $\sim 4$ minutes. This indicates that the saturation coverage of covalently bound aryl moieties is reached at approximately 4 minutes. We note that pristine HOPG samples did not show any D band in their Raman spectra indicating negligible intrinsic density of defects (see Fig. S7 in the ESI†) on the pristine sample.

The AFM images of covalently modified HOPG (Fig. 3c) substrates revealed granular porous films similar to those

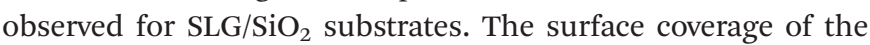
films increases as a function of the reaction in line with the trend observed in the Raman data i.e. it increases with an increase in the reaction time initially and then saturates at longer reaction times. The pits observed in the covalent films a)

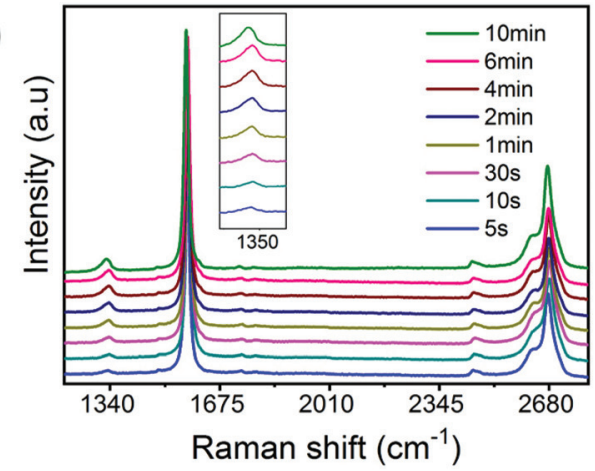

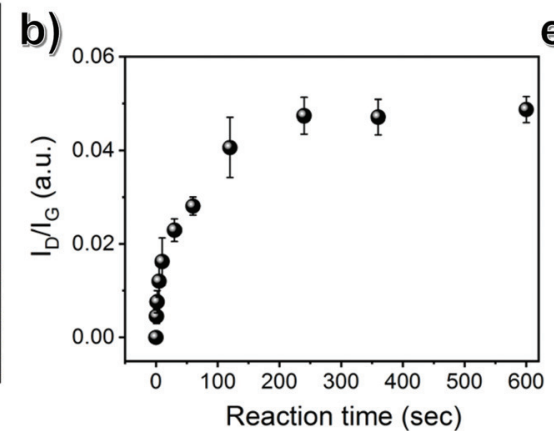

e)

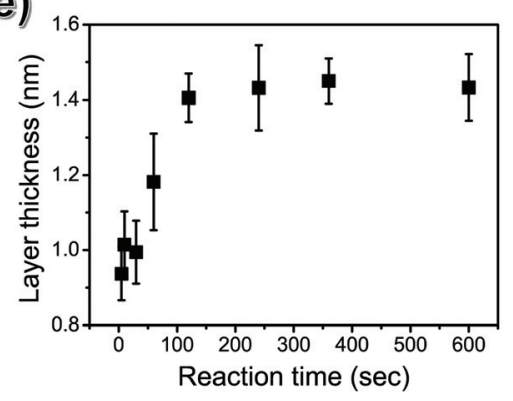

25

30

35

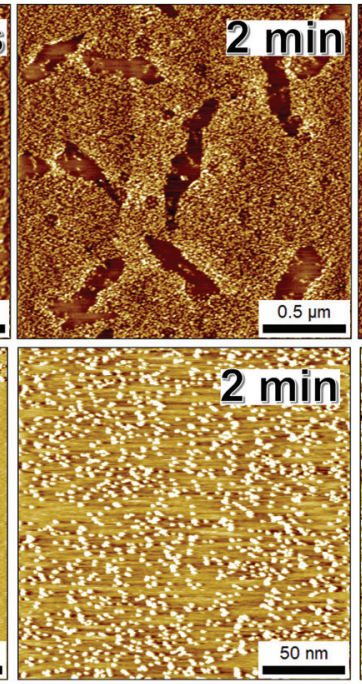

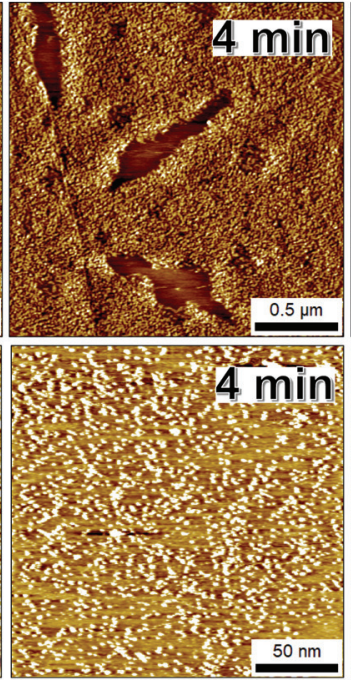

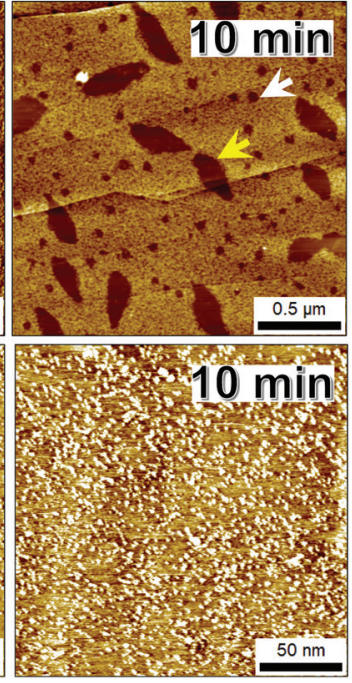

Fig. 3 Dependence of reaction time on covalent grafting of NBD on the surface of HOPG. [KI] $=10 \mathrm{mM}$, [NBD] = $10 \mathrm{mM}$. (a) Raman spectra showing the evolution of the $\mathrm{D}$ band $\left(\sim 1340 \mathrm{~cm}^{-1}\right)$ as a function of reaction time. (b) Variation in the $I_{\mathrm{D}} / I_{\mathrm{G}}$ ratio with reaction time. Representative AFM (c) and STM (d) images showing the evolution of the surface morphology as a function of the reaction time. The white and the yellow arrows in the AFM image (reaction time $=10$ minutes) highlight the circular and elongated regions which represent unmodified areas left on the surface after the reaction. Imaging parameters (STM): $I_{\text {set }}=60 \mathrm{pA}-100 \mathrm{pA}, V_{\text {bias }}=-600 \mathrm{mV}$ to $-800 \mathrm{mV}$. (e) Variation in the layer thickness obtained from AFM data versus the reaction time. (Additional STM/AFM data: see Fig. S6 in the ESI. $\dagger$ ) 
1 plausibly represent unmodified regions where covalent grafting did not take place. These regions are either circular or elongated in shape (see Fig. 3c, reaction time $10 \mathrm{~min}$ ). These empty regions allow straightforward estimation of the layer thickness from AFM height measurements (Fig. S8 in the ESI $\dagger$ ). We note that the thickness measured by scratching the film with the AFM tip in contact mode matches closely with that measured across such empty areas (Fig. S9 in the ESI $\dagger$ ) indicating that they are indeed 'empty'. Fig. 3e shows the changes in the layer thickness as a function of reaction time. It increases initially and then reaches a limiting thickness of $1.4 \mathrm{~nm}$.

Comparison of the variation in the layer thickness with that for $I_{\mathrm{D}} / I_{\mathrm{G}}$ versus the reaction time provides an insight into the details of the interfacial grafting process. Given that the height of a nitrophenyl group covalently attached to the basal plane of graphite is $\sim 0.8 \mathrm{~nm},{ }^{29}$ it is clear that the covalent films obtained at shorter reaction times (up to $\sim 1 \mathrm{~min}$ ) indicate formation of a monolayer of nitrophenyl groups. At reaction times longer than $\sim 1$ min however, the $I_{\mathrm{D}} / I_{\mathrm{G}}$ ratio continues to increase indicating gradual increase in the density of $\mathrm{sp}^{3}$ defects which then saturates at $\sim 4 \mathrm{~min}$. The layer thickness on the other hand, also rises till about $\sim 2$ min and then remains constant $(\sim 1.4 \mathrm{~nm})$. Comparison of these two trends indicates that between $\sim 1 \mathrm{~min}$ to $\sim 4 \mathrm{~min}$, the nitrophenyl radicals not only continue to react with the basal plane of graphite but they also lead to polyaryl growth via reaction with surface-attached nitrophenyl groups. For reaction times longer than $\sim 4 \mathrm{~min}$, the nitrophenyl radicals almost exclusively lead to polyaryl growth by reacting at the sites available on the already grafted layer.

The samples were also characterized using STM. Representative STM data presented in Fig. 3d shows bright features on HOPG samples treated with the reaction mixture only for 5 seconds. We assign these bright features to covalently attached aryl groups. ${ }^{16-18}$ Similar to the trend observed in the Raman and the AFM data, the density of covalent grafting increases with increasing reaction time initially, and then appears to saturate for longer reaction times.

The saturation observed in the thickness of the grafted layer after $\sim 4$ min is rather puzzling. Given the availability of the nitrophenyl radicals, the layer thickness should continue to increase. However, it levels off at $\sim 1.4 \mathrm{~nm}$. In fact, increasing the reaction time to 30 minutes while keeping the same concentration of reagents $([\mathrm{NBD}]=[\mathrm{KI}]=10 \mathrm{mM})$ did not lead to any increase in the layer thickness (Fig. S10 in the ESI $\dagger$ ). These results could be understood by considering the depletion of reactive species in solution via reaction with the surface, the grafted layer, and side reactions which inevitably transpire during decomposition of diazonium salts (also see Fig. 6a). This hypothesis is further supported by concentration-dependent measurements described below where the layer thickness continues to rise for concentrations higher than $10 \mathrm{mM}$ and reaches up to $2.6 \mathrm{~nm}$.

We note that drop casting a $10 \mathrm{mM}$ solution of NBD alone on the HOPG surface did not yield any covalently grafted films. STM and Raman data provided in Fig. S11 in the ESI $\dagger$ revealed no features indicating of covalent grafting as well as an absence of the D-band, respectively. These observations further confirm the significant increase in the efficiency of the grafting process via iodide mediated reduction of NBD in comparison to spontaneous grafting.

Influence of the concentration of the reagents. To gain more insight into the covalent functionalization process, we systematically explored the influence of the concentration of the two reagents on the efficiency of the process and the film morphology. For these experiments, the reaction time (10 $\mathrm{min})$ as well as the molar ratio of NBD and KI (1:1) were kept constant. Fig. 4a shows the evolution in the Raman spectra of the modified substrates as a function of concentration. A noticeable D band appears only at concentrations of around $3 \mathrm{mM}$ and its intensity continues to increase with increasing concentration. At concentrations higher than $20 \mathrm{mM}$, the intensity of the $\mathrm{D}$ band appears to decrease slightly. A clearer inference can be drawn from the plot of $I_{\mathrm{D}} / I_{\mathrm{G}}$ versus concentration (Fig. 4b) which reveals an initial increase in the density of $\mathrm{sp}^{3}$ defects till $\sim 20 \mathrm{mM}$, followed by saturation and then a decrease at concentrations higher than $30 \mathrm{mM}$.

The AFM data presented in Fig. 4c show the changes in the morphology as a function of concentration. Similar to the time evolution experiments described earlier, the surface coverage of the covalent films increases with increasing concentration as evident from the AFM data. Fig. 4e shows that the layer thickness increases almost linearly with an increase in the concentration. Once again, the comparison of the trends for $I_{\mathrm{D}} / I_{\mathrm{G}}$ values and the layer thickness provide important insight into the film growth. The two trends are clearly different and the mismatch observed in these two trends indicates that the major contribution to the film thickness after $\sim 20 \mathrm{mM}$ comes from polyaryl growth. The layer thickness for the highest concentration studied here reaches $\sim 2.6 \mathrm{~nm}$ indicating significant growth orthogonal to the surface. Additionally, the decrease in the $I_{\mathrm{D}} / I_{\mathrm{G}}$, which indicates a decrease in the number of $\mathrm{sp}^{3}$ defects and thus a decrease in the efficiency of grafting with an increase in the concentration could be a result of multiple factors such as increase in side reactions in solution and/or increased extent of polyaryl growth which is clearly reflected in the layer thickness and also increase in the unmodified regions (nanocorrals) (also see Fig. S13 in the ESI $\dagger$ ).

Influence of the mole ratio of the reagents. Variation in the mole ratio of the two components also produced similar trends as those observed for concentration dependence. In these experiments, the concentration of NBD $(10 \mathrm{mM})$ and the reaction time (10 $\mathrm{min}$ ) were kept constant while the concentration of KI was increased resulting in a series of $[\mathrm{KI}] /[\mathrm{NBD}]$ mole ratios ranging from 0.01 to 10 . Fig. 5a shows that a noticeable D band appears only at a mole ratio of 0.5 which first increases in intensity and then appears to decrease as the mole ratio increases. The $I_{\mathrm{D}} / I_{\mathrm{G}}$ ratio shows a rather steep increase till $[\mathrm{KI}] /[\mathrm{NBD}]=1$ and then decreases drastically with a further increase in the mole ratio (Fig. 5b). The average layer thickness of the covalent film increases gradually at lower 
a)

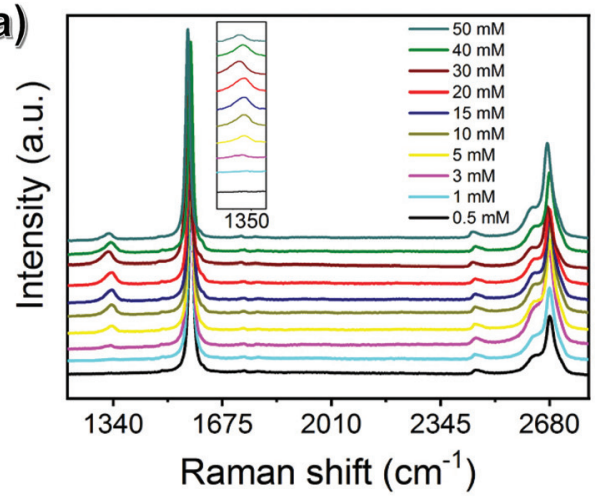

c)

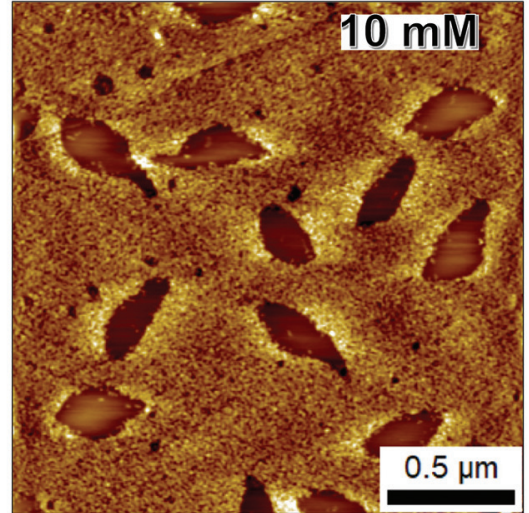

b)
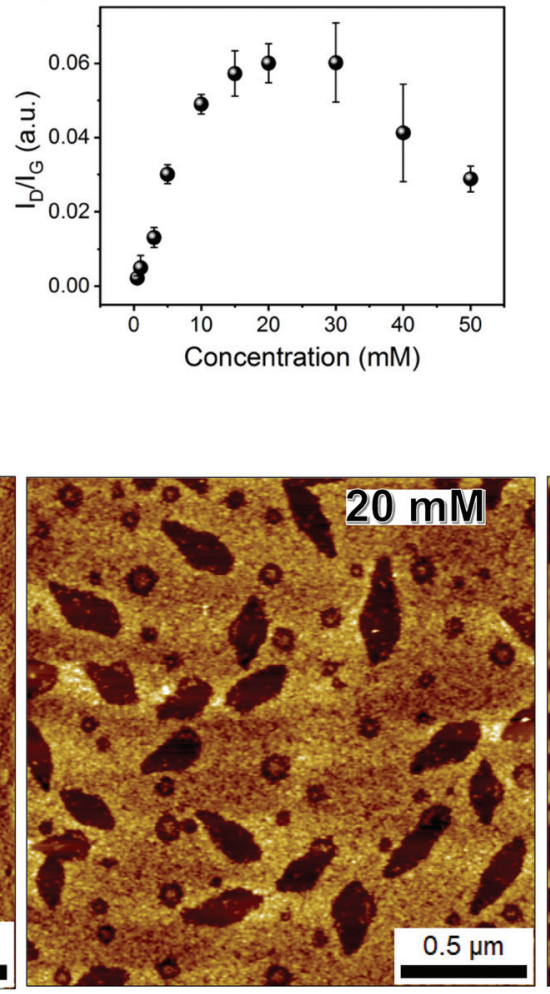

d)
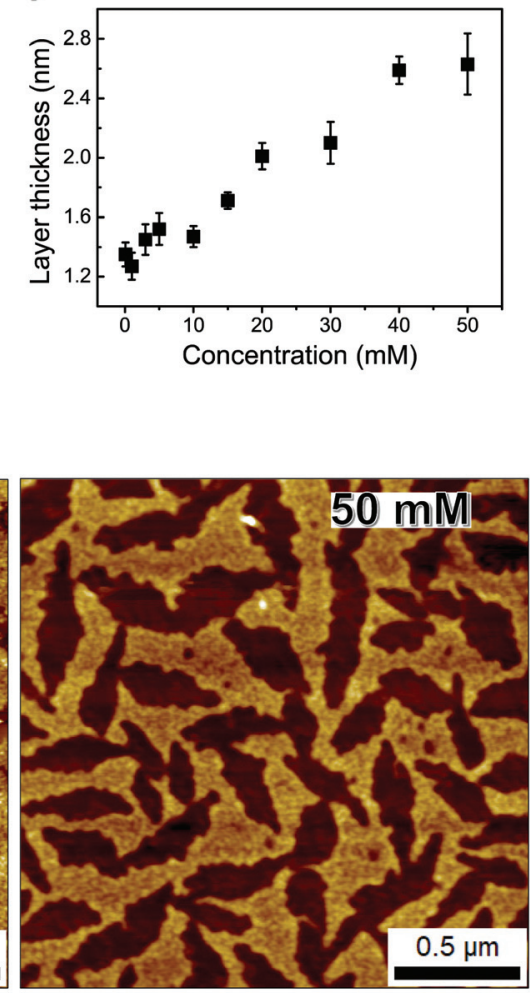

1

5

Fig. 4 Dependence of concentration on covalent grafting of NBD on the surface of HOPG. [NBD]/[KI] = 1:1 and reaction time = 10 min. (a) Raman spectra showing the evolution of the $D$ band $\left(\sim 1340 \mathrm{~cm}^{-1}\right)$ as a function of solution concentration. (b) Variation in the $I_{D} / I_{G}$ ratio with concentration. (c) Representative AFM images showing the evolution of the surface morphology as a function of the concentration. (d) Variation in the layer thickness obtained from the AFM data as a function of concentration. For STM and additional AFM data see Fig. S14 in the ESI. $\dagger$

mole ratios followed by a steep increase at higher mole ratios. Given the significant drop observed in $I_{\mathrm{D}} / I_{\mathrm{G}}$ after the mole ratio reaches 1 , the increase in the layer thickness could be ascribed to polyaryl growth as described earlier. The decrease in $I_{\mathrm{D}} / I_{\mathrm{G}}$ in turn, could be understood by considering both polyaryl growth and the slight increase in non-grafted areas as evident from AFM data. Some AFM images especially those corresponding higher mole ratios (see for example, mole ratio 5 and 10 in Fig. 5c) show presence of a deposit inside the nongrafted regions. Such deposits (height $\sim 0.6 \mathrm{~nm}$, Fig. S9c and d in the ESI $\dagger$ ) are observed randomly in different samples and could arise from physisorbed by-products.

The curious case of nanocorrals. The empty regions observed within the grafted films are peculiar and merit some attention. As described earlier, they are either circular or elongated. The circular ones are typically smaller in size compared to the elongated ones (Fig. 3c) and could originate due to the presence of gaseous nanobubbles accumulated at the hydrophobic HOPG/water interface. ${ }^{30}$ Given that one of the side products of the decomposition reaction used here is nitrogen gas, it is not unreasonable to attribute the circular areas to the presence of nitrogen gas bubbles which occupy the surface and thus prevent covalent attachment of the aryl radicals to the graphite surface. In fact, addition of the reaction mixture to the HOPG surface leads to immediate appearance of gas bubbles which gradually grow in size with increasing reaction time (see Fig. S1 in the ESI $\dagger$ ). These larger bubbles are obviously formed from smaller ones which first nucleate at the HOPG/water interface.

Such interfacial nanobubbles and their stability have been the subject of intense debate over past few years. A number of plausible reasons for the stability of nanobubbles have been proposed including contact line pinning, ${ }^{31}$ organic contaminants, ${ }^{32}$ and dynamic equilibrium between the influx and the outflux of gas at the contact line. ${ }^{33}$ There is an additional hypothesis that the nanobubbles do not adsorb directly on the surface but rather atop a monolayer of adsorbed gas spreading over several hundred nanometres. The nanobubbles and the adsorbed gas layers or the so called 'micropancakes' have been studied using force microscopy and spectroscopy. ${ }^{34}$ While the contamination hypothesis is still being debated, ${ }^{35}$ in the present case, the organic side products formed during the course of the reaction (vide infra) may in fact contribute to the stability of the nanobubbles or alternatively block the surface via physisorption.

It is worth noting that such empty areas are not observed when NBD is subjected to electrochemical grafting. ${ }^{16}$ This suggests that the number of organic by-products formed in 

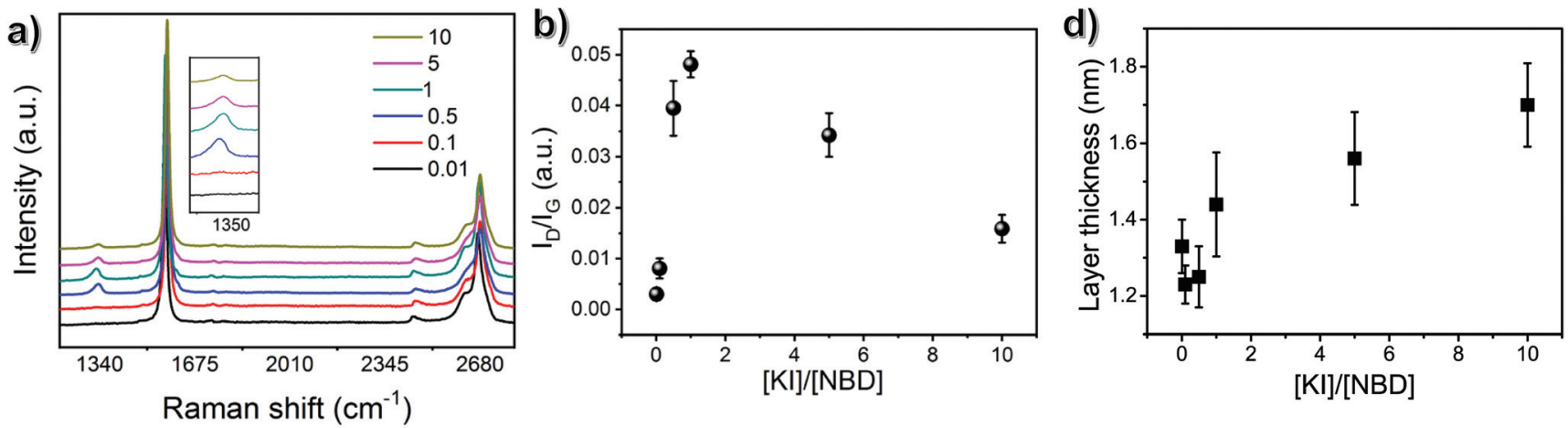

c)
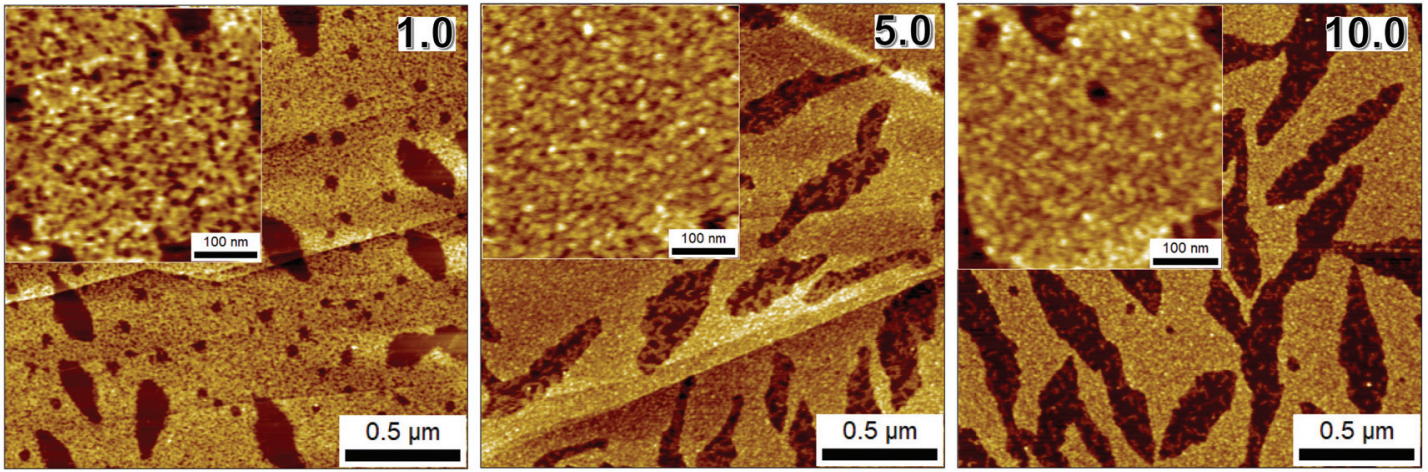

Fig. 5 Dependence of the $[\mathrm{KI}] /[\mathrm{NBD}]$ solution mole ratio on covalent grafting on the surface of HOPG. [NBD] $=10 \mathrm{mM}$ and reaction time $=10 \mathrm{~min}$. (a) Raman spectra showing the evolution of the $\mathrm{D}$ band $\left(\sim 1340 \mathrm{~cm}^{-1}\right)$ as a function of the $[\mathrm{KI}] /[\mathrm{NBD}]$ mole ratio. (b) Variation in the $I_{\mathrm{D}} / I_{\mathrm{G}}$ ratio with mole ratio. (c) Representative AFM images showing the evolution of the surface morphology as a function of the mole ratio. (d) Variation in the layer thickness as a function of mole ratio. solution during the chemical reduction of NBD is relatively higher compared to that during electrochemical reduction. The blocking of the graphene/graphite surface by physisorbed by-products prevents the reaction of the aryl radicals with the surface leading to the unfunctionalized regions. Furthermore, the increase in the surface coverage of unmodified regions with an increase in the concentration of reagents and/mole ratio, which is apparent from the AFM data, is further suggestive of increased by-product formation in solution under those conditions. We have recently used this hypothesis to rationalize the controlled formation of circular nano-pits on the HOPG surface via electrochemical decomposition of a mixture of diazonium salts. $^{27}$

Proposed mechanism and UV-Vis characterization. Having discussed the possible origins of the vacant unmodified areas, we now turn our attention to the mechanistic aspects of the functionalization process. As evident from the scanning probe microscopy and the Raman data presented above, the iodide mediated reductive decomposition of NBD is a fairly complex process wherein the fate of nitrophenyl radicals is dependent on a number of factors. We propose a reaction mechanism presented in Fig. 6a for the KI mediated interfacial decomposition of NBD. The reaction begins with a single electron transfer from the iodide anion to the NBD cation. This leads to the formation of an NBD radical cation and an iodide radical. The NBD radical cation then decomposes via a homolytic cleavage of the carbon-nitrogen bond releasing nitrogen gas and a nitrophenyl radical. The nitrophenyl radical can react in a number of different ways to yield stable products as depicted in Fig. 6a. It can react with the carbon atoms of the graphite lattice forming a carbon-carbon bond with the surface. It can also react with (i) the iodide radical produced in the first step to yield 1-iodo-4-nitrobenzene (INB) (ii) another nitrophenyl radical yielding 4,4'-dinitro-1,1'-biphenyl (DNBP) or (iii) water to yield 4-nitrophenol. ${ }^{36}$ The iodine radical itself can then either combine with another iodine radical to yield molecular iodine which can further react with iodide ions in solution to produce triiodate $\left(\mathrm{I}_{3}{ }^{-}\right)$.

In order to obtain insight into the identity of the reaction products and to shine light on the reaction mechanism, the iodide mediated reductive decomposition of NBD was further studied using UV-Vis spectroscopy. The absorption maxima of the reagents and the possible reaction products mentioned above were first determined (Fig. S15 in the ESI $\dagger$ ). The UV-Vis spectra of the reaction mixture $(\mathrm{NBD}+\mathrm{KI})$ were obtained as a function of solution mole ratio. The positions of the absorption bands in the reaction mixture support that the proposed products are indeed formed during the decomposition process. We note that relatively lower concentrations of NBD and KI were used for the UV-Vis experiments to prevent any aggregate/precipitate formation which ensure applicability of the Beer-Lambert law. The time evolution of the absorption 

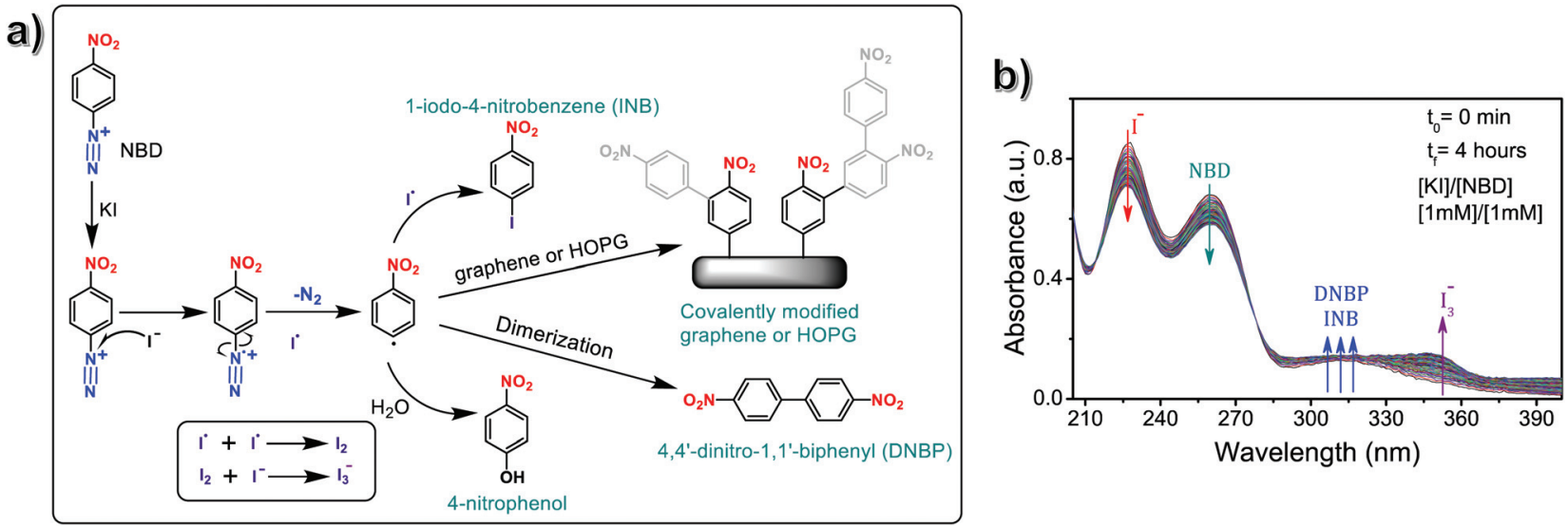

Fig. 6 (a) Proposed mechanism for the arylation of graphene via iodide mediated decomposition of NBD. (b) UV-Vis absorption measurements for monitoring iodide mediated reductive decomposition of NBD in aqueous solutions. The plot shows time-dependent changes in the absorption spectrum of the reaction mixture at room temperature. $[\mathrm{KI}] /[\mathrm{NBD}]=1$.

spectra of the reaction mixture at different mole ratios was monitored (Fig. S16 in the ESI†े).

Fig. 6b shows one such example where the absorption intensities of KI and NBD decrease as a function of time, while absorption peaks at higher wavelengths corresponding to the INB, DNBP and $\mathrm{I}_{3}{ }^{-}$increase. The spectra show a clear isosbestic point at $280 \mathrm{~nm}$. However, the overlap in the absorption spectra above $300 \mathrm{~nm}$ makes it difficult to analyse this region unambiguously. As a consequence, the change in the absorption maximum of NBD $(\sim 260 \mathrm{~nm})$ was used to determine the rate of NBD consumption during the reaction as a function of mole ratio (Fig. S17 in the ESI $\dagger$ ). Detailed analysis of timedependent UV-Vis data presented in the $\mathrm{ESI} \dagger$ revealed the rate of NBD decomposition strongly depends on the amount of KI in solution and it accelerates at higher mole ratios. We note that, in its aqueous solution, the absorbance of NBD does not change significantly over a period of time indicating slower rate of decomposition in the absence of KI (Fig. S18 in the ESI $\dagger$ ). This further confirms the accelerated decomposition of NBD by KI. We note that the proposed mechanism based on the UV-Vis spectroscopy data offers a rather simplified picture. In reality, the functionalization process occurring at the solution/graphene interface may be more complex and thus may require an independent, in-depth analytical characterization of the reaction by-products, which is not within the scope of the current study.

It is worth highlighting the somewhat obvious yet fundamental difference between the electrochemical grafting, the graphenide approach, and the iodide mediated reduction approach presented here. Instead of activating the substrate by applying either electrical potential or by making it reactive by intercalation of $\mathrm{Na} / \mathrm{K}$ ions, the current approach relies on 'activating' the diazonium salts themselves. While in principle, the two strategies can be considered equivalent, the difference lies in the ease of experiment. The iodide mediated covalent grafting can be carried out under ambient conditions, using aqueous solutions and in a matter of seconds. The overall pro- tocol is highly flexible and the degree of covalent grafting can be tuned precisely as demonstrated. As it stands, it is mostly applicable to surface-supported graphene and HOPG. For bulk covalent functionalization of graphene starting from graphite, the graphenide approach still remains superior, as it also allows efficient exfoliation of graphene prior to functionalization. Given that it is combined with mild and efficient exfoliation step, the iodide mediated functionalization approach presented here also holds promise for bulk covalent functionalization of graphene starting from graphite.

\section{Conclusions and outlook}

Thanks to the advent of graphene and the pressing need of its chemical modification for potential applications, the covalent functionalization of carbon-based materials has re-gained popularity. Amongst the strategies used for attaching functional groups to the graphene surface, diazonium chemistry remains the most popular approach. It is used either under electrochemical or environmental control. The iodide mediated decomposition of diazonium salts presented in this work arguably combines the best of both worlds, namely the efficiency of the electrochemical approach and the scalability of the bulk, wet chemical approach.

Our functionalization approach follows a simpler, cleaner and milder protocol that avoids the use of corrosive chemicals and/or long reaction times. It is an efficient strategy for sustainable covalent functionalization of substrate-supported SLG as well as HOPG where the reaction proceeds rapidly at room temperature via simple drop casting of aqueous solutions of the reagents on the substrate. The density of the covalently attached aryl groups as well as the overall thickness of the covalent film could be readily controlled via the reaction time, the concentration, and the stoichiometry of the reagents. Indepth characterization of the functionalized surfaces using scanning probe microscopy and Raman spectroscopy allowed 
us to correlate the layer morphology, the layer thickness and the density of $\mathrm{sp}^{3}$ defects, which in turn disclosed whether the aryl radicals preferentially react with the basal plane of graphite or contribute to polyaryl growth under the given experimental conditions. Further molecular level insight into the reaction mechanism was obtained using UV-Vis absorption spectroscopy.

Besides presenting a mild and efficient approach for covalent functionalization, the present study offers molecular level insight into the interfacial grafting process. Such molecular level insight is critical for the optimization of film thickness and grafting densities. A number of aspects however still remain under investigation. The empty regions formed on the surface of modified substrates reduce the total functionalized area. Assuming that the nanobubbles formed by nitrogen gas is a possible reason for such non-grafted regions, this may be a generic issue associated with the chemical reduction of diazonium salts for such functionalization. As mentioned earlier, such empty areas, if formed with controlled density, could be useful for surface patterning and for studying self-assembly under nanoconfinement. The strategy could be further optimized for more diazonium salts so that a wide library of functional groups could be attached to the basal plane of graphene using the current approach. Lastly, a combination of this approach with an in situ diazotization step would allow the use of aryl amines instead of diazonium salts, which would greatly widen the scope of this approach. These outstanding issues are the subject of an ongoing investigation.

\section{Conflicts of interest}

There are no conflicts to declare.

\section{Acknowledgements}

We thank Dr Miriam Candelaria Rodríguez González and Dr Joan Teyssandier for carefully reading and commenting on the manuscript. The authors gratefully acknowledge financial support from the Fund of Scientific Research Flanders (FWO), KU Leuven - Internal Funds. This work was in part supported by FWO under EOS 30489208. The research leading to these results has also received funding from the European Research Council under the European Union's Seventh Framework Programme (FP7/2007-2013)/ERC Grant Agreement No. 340324 to S. D. F. Y. X. acknowledges financial support through the China Scholarship Council (CSC). C. M. thanks financial support from FWO (grant numbers 12J1719N, 12J1716N). J. S. acknowledges financial support through a Marie SkłodowskaCurie Individual Fellowship (EU project 789865-EnSurf). W. T. and S. E. acknowledge financial support through the Accelerate3 project from the Interreg Vlaanderen-Nederland program, Flanders Innovation \& Entrepreneurship, and the Province of West-Vlaanderen, as well as from KU Leuven internal grant 3E180424.

\section{Notes and references}

1 G. Bottari, M. Á. Herranz, L. Wibmer, M. Volland, L. Rodríguez-Pérez, D. M. Guldi, A. Hirsch, N. Martín, F. D'Souza and T. Torres, Chem. Soc. Rev., 2017, 46, 44644500.

2 V. Georgakilas, M. Otyepka, A. B. Bourlinos, V. Chandra, N. Kim, K. C. Kemp, P. Hobza, R. Zboril and K. S. Kim, Chem. Rev., 2012, 112, 6156-6214.

3 J. J. Navarro, S. Leret, F. Calleja, D. Stradi, A. Black, R. Bernardo-Gavito, M. Garnica, D. Granados, A. L. Vázquez de Parga, E. M. Pérez and R. Miranda, Nano Lett., 2016, 16, 355-361.

4 L. Valenta, P. Kovaříček, V. Valeš, Z. Bastl, K. A. Drogowska, T. A. Verhagen, R. Cibulka and M. Kalbáč, Angew. Chem., Int. Ed., 2019, 58, 1324-1328.

5 M. Delamar, R. Hitmi, J. Pinson and J. M. Saveant, J. Am. Chem. Soc., 1992, 114, 5883-5884.

6 S. Mahouche-Chergui, S. Gam-Derouich, C. Mangeney and M. M. Chehimi, Chem. Soc. Rev., 2011, 40, 4143-4166.

7 F. Barrière and A. J. Downard, J. Solid State Electrochem., 2008, 12, 1231-1244.

8 E. Bekyarova, M. E. Itkis, P. Ramesh, C. Berger, M. Sprinkle, W. A. de Heer and R. C. Haddon, J. Am. Chem. Soc., 2009, 131, 1336-1337.

9 K. C. Knirsch, R. A. Schäfer, F. Hauke and A. Hirsch, Angew. Chem., Int. Ed., 2016, 55, 5861-5864.

10 A. Pénicaud and C. Drummond, Acc. Chem. Res., 2013, 46, 129-137.

11 G. Abellán, M. Schirowski, K. F. Edelthalhammer, M. Fickert, K. Werbach, H. Peterlik, F. Hauke and A. Hirsch, J. Am. Chem. Soc., 2017, 139, 5175-5182.

12 T. Wei, M. Kohring, M. Chen, S. Yang, H. B. Weber, F. Hauke and A. Hirsch, Angew. Chem., 2020, 59, 56025606.

13 A. Criado, M. Melchionna, S. Marchesan and M. Prato, Angew. Chem., Int. Ed., 2015, 54, 10734-10750.

14 M. Z. Hossain, M. A. Walsh and M. C. Hersam, J. Am. Chem. Soc., 2010, 132, 15399-15403.

15 C.-J. Shih, Q. H. Wang, Z. Jin, G. L. C. Paulus, D. Blankschtein, P. Jarillo-Herrero and M. S. Strano, Nano Lett., 2013, 13, 809-817.

16 J. Greenwood, T. H. Phan, Y. Fujita, Z. Li, O. Ivasenko, W. Vanderlinden, H. Van Gorp, W. Frederickx, G. Lu, K. Tahara, Y. Tobe, H. Uji-i, S. F. L. Mertens and S. De Feyter, ACS Nano, 2015, 9, 5520-5535.

17 K. Tahara, Y. Kubo, B. Lindner, S. Hashimoto, S. Hirose, A. Brown, B. Hirsch, L. Daukiya, S. De Feyter and Y. Tobe, Langmuir, 2019, 35, 2089-2098.

18 K. Tahara, T. Ishikawa, B. E. Hirsch, Y. Kubo, A. Brown, S. Eyley, L. Daukiya, W. Thielemans, Z. Li, P. Walke, S. Hirose, S. Hashimoto, S. De Feyter and Y. Tobe, ACS Nano, 2018, 12, 11520-11528.

19 K. Tahara, Y. Kubo, S. Hashimoto, T. Ishikawa, H. Kaneko, A. Brown, B. E. Hirsch, S. D. Feyter and Y. Tobe, J. Am. Chem. Soc., 2020, 142, 7699-7708. 
20 J. J. Navarro, F. Calleja, R. Miranda, E. M. Pérez and A. L. Vázquez de Parga, Chem. Commun., 2017, 53, 1041810421.

21 F.-X. Felpin and S. Sengupta, Chem. Soc. Rev., 2019, 48, 1150-1193.

22 D. Kosynkin, T. M. Bockman and J. K. Kochi, J. Am. Chem. Soc., 1997, 119, 4846-4855.

23 A. C. Ferrari and D. M. Basko, Nat. Nanotechnol., 2013, 8, 235-246.

24 S. Niyogi, E. Bekyarova, M. E. Itkis, H. Zhang, K. Shepperd, J. Hicks, M. Sprinkle, C. Berger, C. N. Lau, W. A. deHeer, E. H. Conrad and R. C. Haddon, Nano Lett., 2010, 10, 40614066.

25 Q. H. Wang, Z. Jin, K. K. Kim, A. J. Hilmer, G. L. C. Paulus, C.-J. Shih, M.-H. Ham, J. D. Sanchez-Yamagishi, K. Watanabe, T. Taniguchi, J. Kong, P. Jarillo-Herrero and M. S. Strano, Nat. Chem., 2012, 4, 724-732.

26 F. Varchon, R. Feng, J. Hass, X. Li, B. N. Nguyen, C. Naud, P. Mallet, J. Y. Veuillen, C. Berger, E. H. Conrad and L. Magaud, Phys. Rev. Lett., 2007, 99, 126805.
27 T. H. Phan, H. Van Gorp, Z. Li, T. M. Trung Huynh, 1 Y. Fujita, L. Verstraete, S. Eyley, W. Thielemans, H. Uji-i, B. E. Hirsch, S. F. L. Mertens, J. Greenwood, O. Ivasenko and S. De Feyter, ACS Nano, 2019, 13, 5559-5571.

28 P. Doppelt, G. Hallais, J. Pinson, F. Podvorica and S. Verneyre, Chem. Mater., 2007, 19, 4570-4575.

29 P. A. Brooksby and A. J. Downard, Langmuir, 2004, 20, 5038-5045.

30 M. Alheshibri, J. Qian, M. Jehannin and V. S. J. Craig, Langmuir, 2016, 32, 11086-11100.

$31 \mathrm{~J}$. H. Weijs and D. Lohse, Phys. Rev. Lett., 2013, 110, 054501.

32 W. A. Ducker, Langmuir, 2009, 25, 8907-8910.

33 M. P. Brenner and D. Lohse, Phys. Rev. Lett., 2008, 101, 214505.

34 H. Peng, M. A. Hampton and A. V. Nguyen, Langmuir, 2013, 29, 6123-6130.

35 X. Zhang, M. H. Uddin, H. Yang, G. Toikka, W. Ducker and N. Maeda, Langmuir, 2012, 28, 10471-10477.

36 C. Bravo-Díaz, U. Costas-Costas, R. Pazo-Llorente and E. González-Romero, J. Chem. Educ., 2000, 77, 384. 